\title{
Data Science Techniques for COVID-19 in Intensive Care Units
}

\author{
Sergio Muñoz Lezcano*, Fernando Carlos López Hernández, Alberto Corbi Bellot \\ Universidad Internacional de La Rioja, Logroño (Spain)
}

Received 15 July 2020 | Accepted 29 October 2020 | Published 19 November 2020

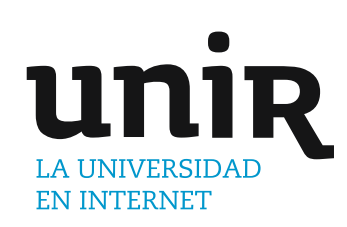

KEYWORDS

COVID-19, Data Science, Machine Learning, Image Processing, Biomarkers, X-Ray, Ventilation.

DOI: $10.9781 /$ ijimai.2020.11.008

\section{IntRoDUCtion}

R Y the end of 2019, doctors in the city of Wuhan (China, province of Hubei) began to detect evidence of the expansion of a new type of severe acute respiratory syndrome (SARS) among the population of the aforementioned city. This event, although different from, had strong similarities to the SARS coronavirus $(\mathrm{CoV})$ outbreak that took place there during 2002-2004, hence the name SARS-CoV-2 or CoVID-19, for short. This new coronavirus is considered to be a strain from the 2002 one and has, by the time of this writing, brought about a novel global pandemic with potentially lethal consequences for infected people. The new disease, COVID-19, was considered as a public health emergency by the World Health Organization (WHO) on January 30, 2020. In March 11, its condition was raised to the status of global pandemic [1].

As the original SARS-CoV coronavirus, SARS-CoV-2 is transmitted through respiratory droplets from person to person and the condition's symptoms appear after an incubation period that lasts from 2 to 14 days [2]. The great expansion rate of the disease has led to a shortage in intensive care equipment and to a greatly increased demand for technical resources (e.g. mechanical ventilation, Personal Protective Equipment (PPE), drug treatments, etc.). In many situations, the health care systems have risked or even surpassed the point of collapse.

This pandemic has also had a huge impact on the generation of clinical and research data. Of course, part of this dataset has been generated in the Intensive Care Units (ICUs) and Emergency Rooms (ERs). However, the exploitation of this information is complex due

\footnotetext{
* Corresponding author.

E-mail address: smunozle@gmail.com
}

to the stressful situations that clinicians experience in ICUs. Some pieces of this data may turn out to be too complex and too varied for obtaining practical and correct diagnoses. In this context, Artificial Intelligence (AI), Image Processing (IP) and Machine Learning (ML) techniques can contribute to manage this problem of information overload. The information flow is represented in Fig. 1, where AI has a pivotal role in the fight against COVID-19 in intensive care units [3].

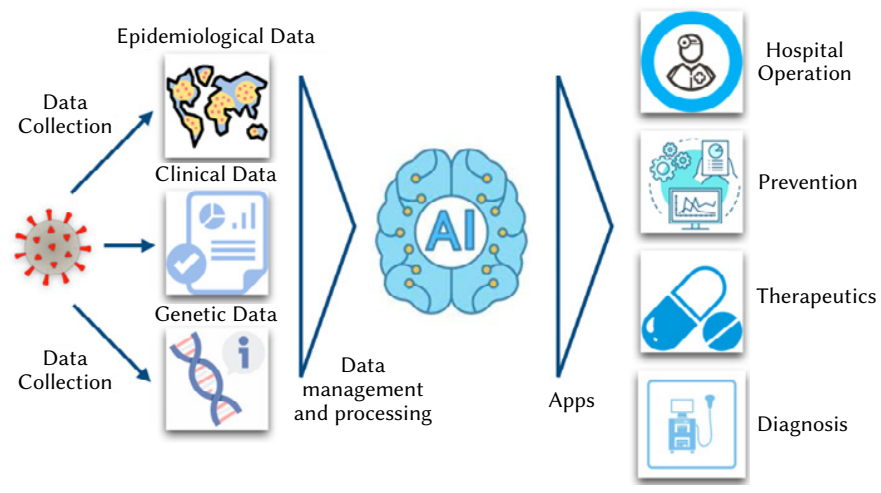

Fig. 1. Areas of interest where artificial intelligence and machine learning could play a key role in the fight against COVID-19 (e.g., biochemistry and clinical data of COVID-19 patients can be studied by ML techniques to focus not only in risk prediction, but also for disease treatment). Model adapted from the figure published [3].

The rest of this paper is organized as follows. First, we review the COVID-19 outbreak, its complexity, symptoms and how healthcare professionals are dealing with ICU patients. Then, we provide a broad prospective on the utility of machine learning in data science. Next we explain how ML is applied in medicine with some examples and 
applications. The next aim is to contextualize the applicability of $\mathrm{AI}+\mathrm{IM}+\mathrm{ML}$ in COVID-19 focusing on its critical areas of interest. Specifically, this review covers mechanical ventilation, medical imaging and clinical data. Finally, we outline the most important findings and prospective future research lines.

\section{The COVID-19 Outbreak}

Dr. Tedros Adhanom Ghebreyesus ${ }^{1}$, the World Health Organization (WHO) Director-General, reported on February 11, 2020 the new Coronavirus Disease SARS-CoV-2 or COVID-19 intensive epidemic in Wuhan. This is the third identified type of dangerous acute respiratory syndrome from coronavirus (SARS-CoV-2) in the last twenty years, but the one with the lowest level mortality rate [4], as evinced in Table I. Until July 13, there were a total of $12,859,834$ confirmed cases, and more than 567.957 deaths in 188 countries and territories around the world ${ }^{2}$. The countries in which the outbreak has had the most severe impact in terms of cases are in Table II: United Stated of America, Brazil, India, Russian Federation, Peru, Chile, Mexico, The United Kingdom, South Africa, Iran, Spain and Pakistan.

TABLE I. CoV MoRTALity RATE

\begin{tabular}{cccc}
\hline CoV & Year & Origin & Mortality Rate \\
\hline SARS & 2002 & Guandong province, China & $10 \%$ \\
MERS & 2013 & Saudi Arabia & $34 \%$ \\
COVID-19 & 2019 & Wuhan, China & $3.4 \%$ \\
\hline
\end{tabular}

Mortality rate comparing different coronavirus outbreaks from 2002 to present

TABLE II. SituATION By Country

\begin{tabular}{cccc}
\hline Country & Cases & Deaths & $\begin{array}{c}\text { Death Rate (\%) of } \\
\text { cases }\end{array}$ \\
\hline United Stated of America & $3,163,581$ & 133,486 & 4.2 \\
Brazil & $1,800,827$ & 70,398 & 3.9 \\
India & 849,553 & 8,196 & 0.9 \\
Russian Federation & 727,162 & 11,335 & 1.5 \\
Peru & 319,646 & 11,500 & 3,6 \\
Chile & 312,029 & 6,881 & 2.2 \\
Mexico & 289,174 & 34,191 & 11.8 \\
The United Kingdom & 288,957 & 44,798 & 15.5 \\
South Africa & 264,184 & 3,971 & 1.5 \\
Iran & 255,117 & 12,635 & 4.9 \\
Spain & 253,908 & 28,403 & 11,2 \\
Pakistan & 248,872 & 5,197 & 2 \\
\hline
\end{tabular}

Globally, as of 4:49 pm CEST, 12 July 2020, there have been 12.552 .765 confirmed cases of COVID-19, including 561.617 deaths, reported by the WHO in https://covid19.who.int.

Scientists think that COVID-19 made a leap from animals to humans as a new zoonotic [5] human disease at the Huanan Seafood Market in Wuhan. In this place, bats, snakes and other wild animals are sold as culinary delicacies. As the Fig. 2 shows, even though the zoonotic root of SARS-CoV-2 has not been corroborated, the series-based analysis indicates that the virus jumped from bats (as this species is an important coronavirus reservoir) to humans perhaps through an intermediate host, the pangolin [6]. Once the virus had the capability of infecting humans, it rapidly spread up to 109 countries in November and December 2019.

\footnotetext{
${ }^{1}$ World Health Organization. Coronavirus press conference 11 February 2020 ${ }^{2}$ Johns Hopkins Coronavirus Resource Center https://coronavirus.jhu.edu/ map.html
}

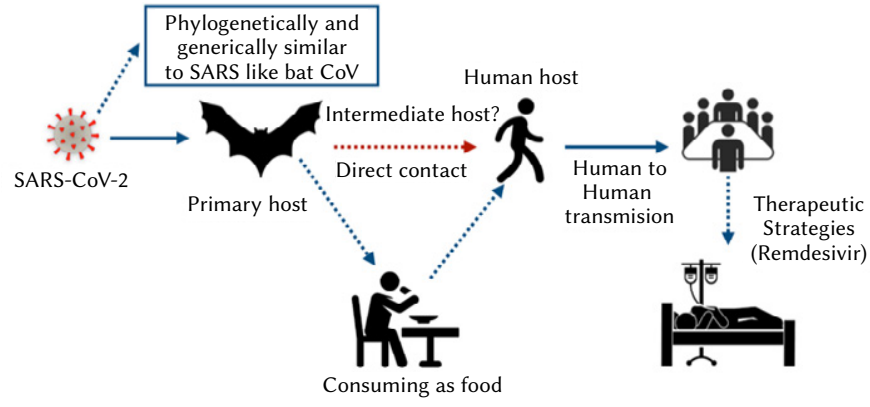

Fig. 2. Representation of the transmission of SARS-CoV-2. Model adapted from the figure published [6].

Direct transmission [7] requires direct physical contact between the infected and predisposed individuals, via bites and handling of infected animal tissue. Direct contact and bites involve the formation of skin rash and ulcerations, whereas the ingestion of infected meat/ water involves digestive tract problems and diseases transmitted through the intake of inhalation of contaminated particles could cause pneumonia.

After a massive outbreak, now driven by human-to-human transmission, the infection continued its expansion thanks to national and migratory movements and global tourism, starting with the Chinese New Year holidays. SARS-CoV-2 is a highly diffusible virus, carried by airborne droplets, direct contact or contact with contaminated objects. Major risk factors are:

- Age greater than 60 (increasing with age).

- Male gender.

- Subjacent non-communicable diseases (NCDs): diabetes, hypertension, cardiac disease, chronic lung disease, cerebrovascular disease, chronic kidney disease, immunosuppression and cancer.

In many cases, the most common symptoms at inception of COVID-19 disease could include [8] -[9]:

- Fever experienced by $83-99 \%$ of the patients [10].

- Neurological manifestations [11] such as anosmia (loss of sense of smell) and dysgeusia (perception of altered taste).

- Dyspnea or shortness of breath (31-40\%).

- Dry cough (59-82\%).

- Fatigue (44-70\%).

- Myalgia (11-35\%).

- Diarrhea.

Moreover the "Massachusetts General Hospital treatment guidance for critically ill patients with COVID-19" [12] reported that it would be expected that a percentage of these patients (5\%) would need ICU admissions due to bilateral pneumonia, acute respiratory distress syndrome (ARDS), hypoxemia, respiratory failure, shock and multiple organ dysfunction syndrome (MODS). As Fig. 3 discloses, critically events lead to further complications [13]. In the most severe cases, a cytokine storm syndrome is triggered that involves an overall hyperinflammation that leads to sepsis and septic shock, thromboembolism, and/or multiorgan failure, that includes in many cases acute kidney injury and cardiac injury. When these events take place, the mortality rate is in the range of $20 \%$ to $60 \%$ after ICU admission. In Wuhan [14], between January 10 and February 29, 2020 at least a median(interquartile range) of 429(25-1143) patients with COVID-19 were reported and admitted in the ICU while 1521 (111-7202) patients were diagnosed with severe sickness each day. During the epidemic peak, 19,425 patients (24.5 per 10,000 adults) were hospitalized, 9,689 (12.2 per 10000 adults) were considered in severe illness, and 2,087 (2.6 per 10,000 adults) needed critical care per day. After the first 7-8 
days in the ICU, patients required massive intensive care management (lung protective ventilation, avoidance of fluid overload and support of organ function) as well as the application and intervention by a wide spectrum of medical technologies and therapies such as:

- ventilators in most cases,

- RT-PCR tests,

- aerosol generating therapies,

- laboratory equipment,

- X-ray and CT Imaging, and

- other healing therapies previously used for SARS and MERS.

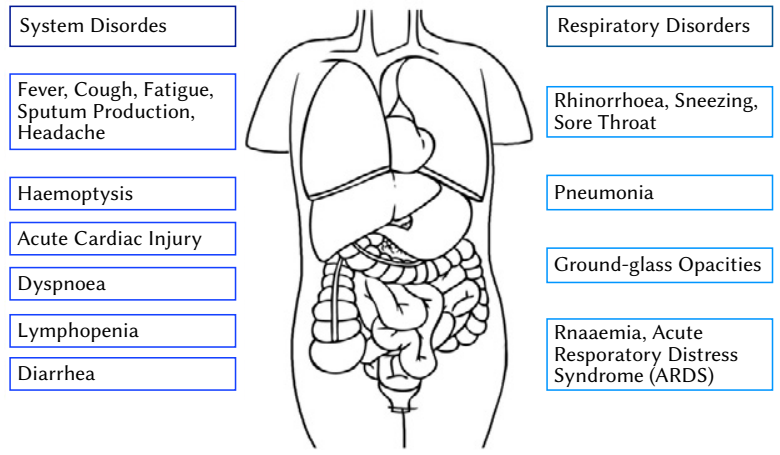

Fig. 3. Severe and abnormal symptoms caused by critical disease complications. e.g. respiratory failure. Model adapted from the figure published [13].

Regarding the use of therapies, even when different drugs have in vitro activity against different coronaviruses, until today there has been no clinical evidence that argues the efficacy and safety of these treatments for coronavirus infection, including SARS-CoV-2. What is more, compassionate treatments that have not been previously approved for clinical purposes could involve serious harmful consequences when taking into account that many patients suffer from previous disorders such as cardiac arrhythmias. In these circumstances, taking into consideration that nonproven specific therapies (NPTs) are available, a large number of patients have received compassionate use therapies such as Remdesivir [15], which had been previously targeted at fighting against the Ebola virus, Lopinavir-Ritonavir (an HIV type 1 aspartate protease inhibitor [16]), chloroquine (generally-used antimalarial and autoimmune disease drug [17]) and hydroxychloroquine (an analogue of chloroquine [15]) among other therapies.

Bearing in mind all these concerns, a significant number of doubts still need to be addressed. In summary, until a vaccine or effective treatment is available, the most efficient measure for reducing personto-person transmission of COVID-19 needs to focus on the use of masks, social distancing and hand washing.

\section{MACHINE LEARNING}

In 1959, Arthur Samuel defined machine learning as a "field of study that gives computers the ability to learn without being explicitly programmed" [18]. We might say, very broadly, that machine learning refers to the changes in systems regarding all those tasks associated with AI. These tasks have a wide range of functions, from recognition, to diagnosis and prevention. Many techniques in machine learning arrive from the efforts of psychologist to find similarities to biological learning. But psychologists are not the only ones who have contributed to the study and evolution of ML. Other disciplines have also made relevant contributions, such as:

- Brain modeling, which tries to interpret learning phenomena in terms of artificial neural networks.
- Statistical machine learning, which can be considered another instance of artificial learning.

- Adaptive control, which manages processing in real time by the use of mathematical estimations in real time.

- Artificial intelligence, which has been associated with ML from the very beginning, since past actions have a direct effect on the future.

- Evolutionary algorithms, which have proposed certain aspects of biological evolution as learning methods. Genetic algorithms are the most representative of these algorithms.

Traditionally, the training of a ML algorithm has been classified into the three broad categories shown in Fig. 4, which depend on the available data [19].

- Supervised learning, which teaches the computer how to do something and then lets it use its new knowledge to do the task.

- Unsupervised learning, by which the computer learns without instruction and uses this knowledge to structure and find patterns in the dataset.

- Reinforcement learning, by which the computer interacts with a changing ecosystem where it needs to perform a specific action or objective, such as driving an autonomous vehicle.

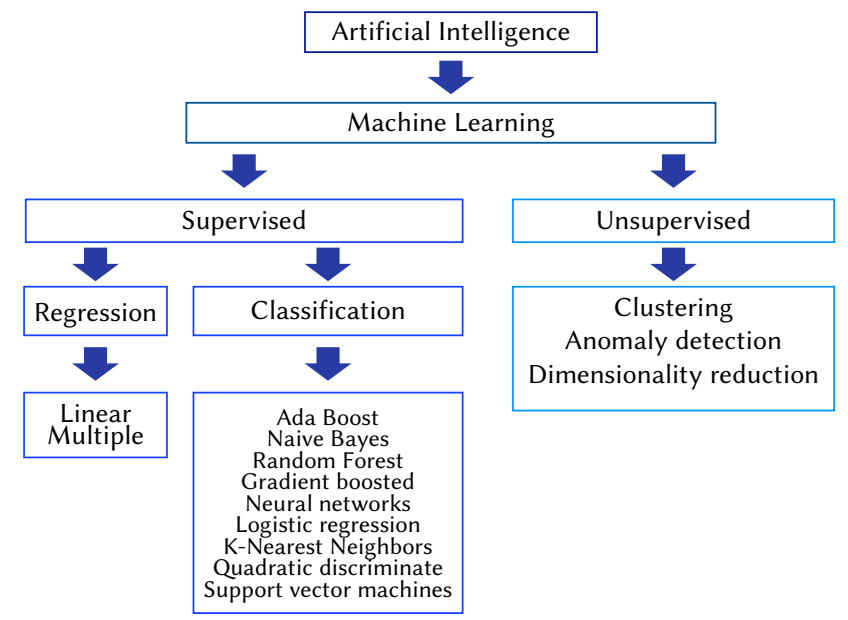

Fig. 4. The major learning approaches in ML. Examples of algorithms of the corresponding type are shown under each branch. Model adapted from the figure published [19].

ML has had an impact on different applied disciplines such as physics, chemistry, engineering, as well as medicine. These techniques can be applied to problems of prediction, classification or control in a wide spectrum of medical fields from the assessment of a patient's state to the prognosis of disease outcome from genetic information.

\section{Machine Learning in Medicine}

The next sections survey machine learning applied to medicine and then focus on its prospective applications for addressing COVID-19, medical imaging, clinical data and the automatic monitoring of ventilators. Until today, experience and heuristics have been the gold standard in medicine, whereby knowledge is acquired through daily practice in self-learning, considering the high variable healthcare environments. Computational resources development as well as data storage capacity has become the big enabler in automatic or semi-automatic data-driven and evidence-based medical decisions. The use of ML in this context relies on the collection and analysis of huge and complex datasets to recognize and learn patterns, limit false 
classifications and make the following decisions related to:

- Diagnosis. Errors in diagnosis happen on a daily basis and this problem is not limited to complex situations. In this scenario, ML could reduce the uncertainty by helping clinicians make better decisions and at the same time reduce errors. In this context, computer-based medical IP (e.g. electrocardiogram, topographies, etc.) enables the non-intrusive identification and medical assessment of the level of damage in different regions of the body.

- Prognosis. ML applications can learn patterns from a vast number of patients. Using data belonging to laboratories for classification of biomarkers, clinicians can anticipate future events going beyond the individual physician's clinical experience and reducing bias.

- Treatment. In a large health care system, there could exist tens of thousands of physicians, and a huge number of patients with related conditions. ML could help clinicians automate, program and at the same time customize treatment in order to apply better treatment pathways. A clear field of application is the assessment of genomic attributes to find personalized treatments.

\section{Artificial Intelligence Applied to Covid-19}

COVID-19 has rapidly become a global concern that needs faster, more sensitive, and at the same time customizable support. Currently, artificial intelligence, in particular ML, can help dealing with the key challenges associated with the SARS-CoV-2 outbreak, which are:

- Diagnostic Capacity. The huge number of cases overwhelms diagnostic potential, accenting the need for an agile pipeline for sample processing and diagnosis.

- Diagnosis Specificity. SARS-CoV-2 is nearly connected to other significant coronavirus subspecies and species, so diagnostic assays can produce false positives if they are not finely specific to SARS-CoV-2.

- Pathogen Characterization. Presumed SARS-CoV-2 patients sometimes suffer from a dissimilar respiratory viral infection and in worst cases have co-infections with SARS-CoV-2 and other respiratory viruses. Consequently, it is important to identify these other pathogens, for both patient diagnostics and pandemic response. For these reasons, the authors in [20] identify (in Table III) an inherent COVID-19 virus genomic signature and compare the performance of their propose algorithm with six machine learning algorithms (Linear Discriminant, Linear SVM, Quadratic SVM, Fine KNN, Subspace Discriminant, and Subspace KNN) for an ultra-rapid adaptable, and extremely accurate classification of whole COVID-19 virus genomes. Table III shows the effectiveness of these approaches.

Taking into account the previously mentioned difficulties, clinicians and nurses need to deal with a vast amount of data, work with multidimensional problems or identify patterns based on a huge dataset. To face this problem, the combination of AI techniques and high-performance computing networks will be the new clinician's tools for health care provision. This new data-driven approach enables decision-taking processes that involve high complexity, are more accurate and with a quicker response, to cure more people when dealing with a lack of resources.

Considering COVID-19 unique circumstances, AI efforts have been mainly focused on ICU activity. Fundamentally, the spotlight has been put on diagnosis, monitoring, prognosis and drug discovery. At the same time, the fast spread of COVID-19 and the lack of resources in all countries have made possible the development of a myriad of applications based on AI to deal with the following clinical problems:

- Patient diagnoses and severity status. Clinical asset restrictions
TABLE III. Classification Accuracy Scores of Betacoronavirus

\begin{tabular}{ccccc}
\hline Dataset & Clusters & $\begin{array}{c}\mathrm{N}^{\circ} \\
\text { Seq }\end{array}$ & $\begin{array}{c}\text { Classification } \\
\text { Model }\end{array}$ & Acc. \\
\hline Test-4: & Embecovirus & 49 & LinearDiscriminant & 97.6 \\
BetaCV; & Merbecovirus & 18 & LinearSVM & 98.4 \\
124 seq.; & Nobecovirus & 10 & QuadraticSVM & 98.4 \\
MaxL:31526 & Sarbecovirus & 47 & FineKNN & 97.6 \\
MinL:29107 & & & SubspaceDiscriminant & 98.4 \\
MdL:30155 & & & SubspaceKNN & 97.2 \\
MnL:30300 & & & AverageAccuracy & 97.6 \\
\hline Tes-5: & Embecovirus & 49 & LinearDiscriminant & 98.6 \\
Beta\&CV19 & Merbecovirus & 18 & LinearSVM & 97.4 \\
152 seq. & Nobecovirus & 10 & QuadraticSVM & 97.4 \\
MaxL:31526 & Sarbecovirus & 47 & FineKNN & 97.4 \\
MinL:29107 & COVID-19 & 29 & SubspaceDiscriminant & 98.7 \\
MdL:29891 & & & SubspaceKNN & 96.1 \\
MnL:30217 & & & AverageAccuracy & 97.5 \\
\hline Test-6: & Sarbecovirus & 47 & LinearDiscriminant & 100 \\
Sarb\&CV19 & COVID-19 & 29 & LinearSVM & 100 \\
76 seq.; & & & QuadraticSVM & 100 \\
MaxL:30309 & & & FineKNN & 100 \\
MinL:29452 & & & SubspaceDiscriminant & 100 \\
MdL:29478 & & & SubspaceKNN & 100 \\
MnL:29772 & & & AverageAccuracy & 100 \\
\hline
\end{tabular}

Relationship between viral sequences, taking into account different levels of taxonomies and the score of Classification's accuracy.

(ICU equipment, etc.) imply that the treatments depend on the seriousness of the patient's status. Since asymptomatic patients could become seriously or even critically ill very fast, it is critical to identify them as soon as possible and administer the right treatment in order to reduce mortality. AI techniques can help to support the analysis, prediction and treatment of COVID-19 infections. Until now, AI applications based on COVID-19 clinical needs have been focused on diagnosis relying on medical imaging to analyze computer tomography (CT) and scans [21]. Authors in [22] and [23] have demonstrated that $\mathrm{ML}+\mathrm{CT}$ or $\mathrm{ML}+\mathrm{X}$-ray combinations give extra time to radiologists for COVID-19 diagnoses, as shown in Fig. 5. Results from this research demonstrate, that ML+CT combinations are quicker and less expensive than the Reverse Transcription Polymerase Chain Reaction (RT-PCR or) test; the current gold standard for COVID-19 diagnosis. This approach makes more sense due to test global shortage and expense of the PCRs. However, not all ICUs have X-ray equipment (or even CT scanners). With the assistance of deep learning techniques, authors in [23] propose classifying the patient status as either negative or positive for COVID-19. This research has reported a $94.92 \%$ sensibility and $92.00 \%$ specificity in COVID-19 detection. These results demonstrate the utility of deep learning models to classify COVID-19 medical imaging.

- Patient's health progress. Patients' clinical data and blood tests contain many analyzable features that help doctors in recognizing high-risk patients [24], as explained in the flowchart in Fig. 6. The sooner the patient is tested, the sooner the prognosis improves. Taking into account this premise, a patient's health status can improve, and the mortality can decline for people seriously sick. So far, this methodology facilitates the interpretability of the most remarkable elements regarding the physiological progress of COVID-19, specifically in cell injury, cell immunity, and tissue inflammation. 


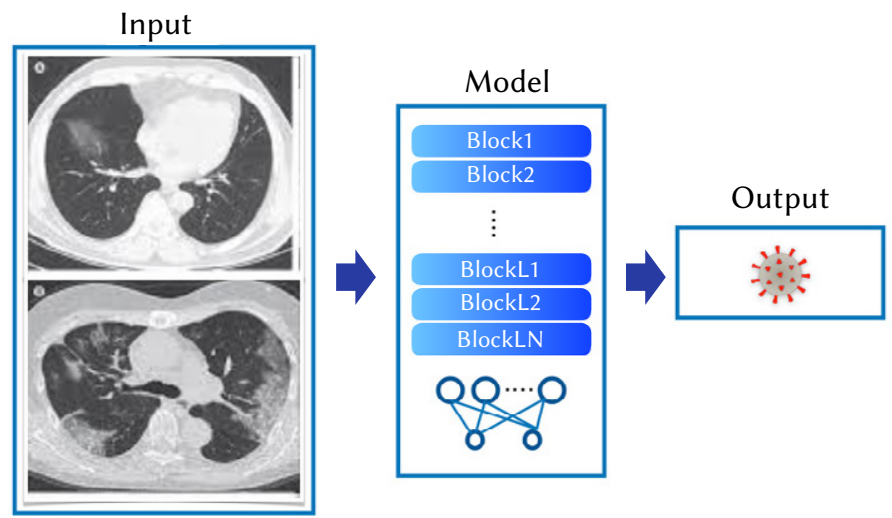

Fig. 5. Deep learning algorithm framework. For each patient's computed tomography scan, ROI (regions of interest) have been randomly selected and used in the inception network to extract features and then make a prediction. Model adapted from the figure published [21].

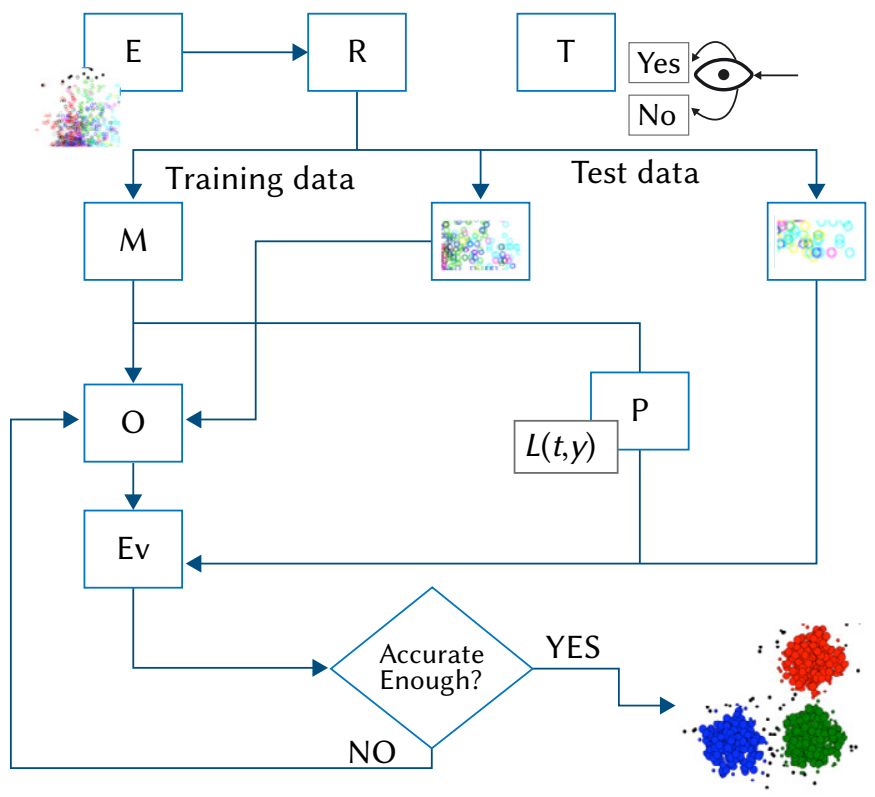

Fig. 6. The flow chart represents different concepts involved in a typical supervised ML process. "E" means experience (data); "R", is related to the task; "T", the classifying task; "M", represents the ML Model (the ML algorithm or the classifier); "O", is the optimization phase; "P", is related to metrics; "Ev", is in charge of the evaluation, here represented as a "loss function" $L(t, y)$. The final feedback involves the "learning" by the machine. Model adapted from the figure published [24].

- Computational biology and drug discovery. This approach is based on disease dynamic modeling [25]. It includes the use of data analytics, mathematical modeling and computational simulation procedures to study the COVID-19 biology. Authors in [26] propose a new pathway that contributes to the understanding of the effect of parameters that govern the proliferation of the disease. The results, as Fig. 7 shows, put the focus on a new AI algorithm that has been designed for identifying drugs that might block the viral infection process. In this way, Baricitinib [26] could decrease the potential of the virus to cause infection in lung cells.

Taking into account this enormous number of combinations, the scenario of COVID-19 gets more complex especially in ICUs with COVID-19 patients. Electronic health records from patients admitted at ICUs has been growing exponentially during the past months.

Clear examples of this new scenario are common ICU equipment such as pressure and flow transducers, infusion pumps, pulse

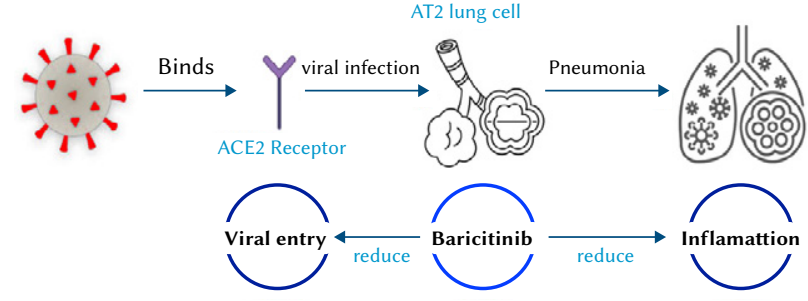

Fig. 7. The receptor ACE2 might be used by 2019-nCoV to infect lung AT2 alveolar epithelial cells. Baricitinib could be appropriate for patients with 2019-nCoV acute respiratory disease in order to reduce both the viral entry and the inflammation in patients caused by cytokines. Model adapted from the figure published [26].

oximeters, cardiac output monitors, and mechanical ventilators. This medical equipment stores data records and includes interoperable interfaces that allow for their interconnection using standards such as the Health Level Seven ${ }^{3}$ (HL7). Through HL7, ICU medical devices communicate with a hub of electronic systems through busses and interchangeable, hot pluggable interfaces.

\section{A. Adaptive Ventilation Settings}

In this section, we focus on ventilation, medical imaging and clinical data topics that seem to be the most relevant areas where the combination of AI and COVID-19 promises better results.

Mechanical ventilation has saved many lives since it was implemented in the 1950 s to tackle poliomyelitis. From that time, the use of ventilation support has been intensified with the past of the years. This increase has been directly associated with the evolution of critical care units [27]. The most important goal of mechanical ventilation (Table IV) is to enhance alveolar gas exchange. Taking into consideration this purpose, the ventilation system is composed of three elements:

- the ventilator together with its inspiratory and expiratory circuits,

- the patient,

- the interface between these two that controls the gas exchange.

TABLE IV. OBjectives of Mechanical Ventilation
Make better pulmonary gas Exchange
Reverse hypoxemia

Improve acute respiratory acidosis

\begin{tabular}{cc} 
Improve acute respiratory acidosis \\
\hline Alleviate respiratory distress & $\begin{array}{c}\text { Reduce oxygen cost of breathing } \\
\text { Revoke respiratory-muscle fatigue }\end{array}$ \\
\hline Modify pressure-volume relations & Prevent and reverse atelectasis \\
Improve Compliance \\
Prevent further injury
\end{tabular}

Allow lung and airway healing

Avoid complications

Main and secondary targets

The ventilation process is simple. In fact, it is the product of the respiratory frequency and the lung volume. This process is defined as the units of ventilation with gas inhaled or exhaled from a person's lungs per minute. Even when it is an apparently simple relationship, ventilation is related with a biological process and for this reason there are always complexities. For instance, the changes in the inspiratory and expiratory dynamics change the mean inspiratory output in

${ }^{3}$ https://www.hl7.org 
volume-preset ventilators, the time for filling the alveolus with gas, the presence of airflow difficulties and finally expiratory volume. Moreover, mechanical ventilation without spontaneous breathing leads to respiratory muscle atrophy. For this reason, clinicians prefer assisted modes initiated by patients' respiratory activity. The most usual procedures are:

- Assist-control ventilation: the ventilator provides a breath independently when triggered by a patient's respiratory activity or not.

- Intermittent mandatory ventilation: the patient gets regular positive pressure breaths from the ventilator at a preset volume and rate. Spontaneous breath is also allowed.

- Pressure-support ventilation: the clinician sets an initial level of pressure (instead of volume) to increase the spontaneous breathing activity by the patient. Airway pressure is preserved at a preset level until the patient's inspiratory flow descends below a certain level.

As indicated in chapter II of this review, COVID-19 disease involves, in many cases, ICU admissions due to bilateral pneumonia, ARDS, hypoxemia, respiratory failure, shock and MODS. These situations require in most cases mechanical ventilation and intubation for helping the patient. Recent studies based on public data from COVID-19 patients from England, Wales, and Northern Ireland, reported that until March 24, 2020, two thirds of patients (i.e., 132 people) admitted at ICU required mechanical ventilation [28]. In most cases, with the shortage of advanced ventilators, the ventilation process needs a manual setup process before they can operate. In this situation, the patient's healing depends on the clinician's ability to modify the patient's respiration. Therefore, it is important to take into consideration the following questions for optimally managing the ventilation support:

- What are the clinical aims?

- Is there a complete comprehension of the foundations and functional limitations of the medical devices?

- What is the medical intention in changing the patient's prescription?

In many cases, there aren't any homogenous treatments, and ventilation involves a huge degree of personalization, so clinical bias takes place. Even when ventilators work in a proper way in delivering air to the lungs, the design of this equipment is based (in many cases) on an open loop flow system, which implies patient having to adapt to the machine. This means that the input ventilation does not depend on the outflow so the patient's response to the flow and the delivered breath can't be assessed. The Fig. 8 represents this open loop flow process.

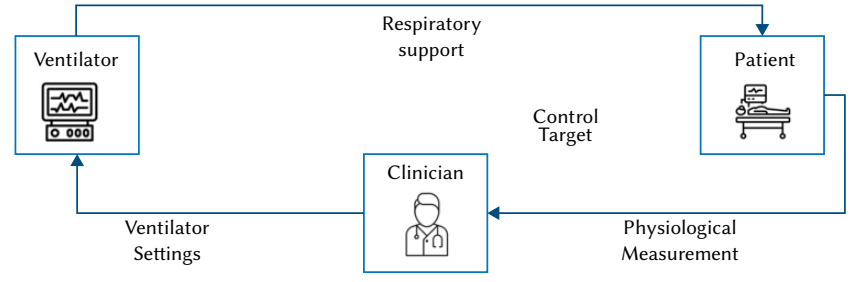

Fig. 8. Open Loop Ventilators Systems.

In order to avoid this issue, automated closed-loop systems (with feedback control) allow doctors to keep a patient at a specified clinical target without permanent clinical interaction. This means that a centralized module, the controller, takes the control over the task of adapting ventilator settings. Fig. 9 represents the closed loop flow.

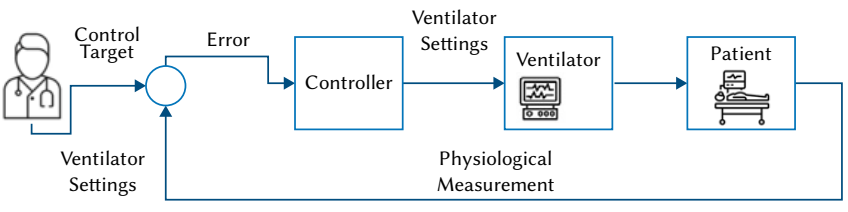

Fig. 9. Closed Loop Ventilators Systems.

From this point of view, machine learning plays a key role in automatic adaptation to customized patient ventilation. Gutierrez [19] mentions various applications regarding the development of AI artifacts that could help with the design of autonomous ventilators, making feasible the continuous monitoring of the ventilation process feasible. These artifacts could adjust some parameters automatically, such as asynchrony in breathing, which is one of the main problems with patient intubation. These techniques could improve advanced modes of closed loop ventilation systems, which imply sharing of information between the ventilator and the patient. For this purpose, the authors of [28] have described one of the most advanced algorithms. This research has compared ML predictions by working with a 1024 breaths' selection based on random criteria that take into account 16 patients. The results were compared to those made by five experts. The assessment reported very good results with sensitivity of $91.5 \%$, specificity of $91.7 \%$, positive predictive value (PPV) of $80.3 \%$ and negative predictive value (NPV) of $96.7 \%$. Additionally, 1,378 breath cycles from 11 mechanically ventilated critical care patients took part on studies related to patient ventilator asynchrony [29]. In this paper, the use of random forest algorithms permitted the detection of the presence or absence of cycling asynchrony with valuable results. In this way, three different situations such as delayed termination, premature termination and no cycling asynchrony were analyzed. The sensitivity (specificity) was respectively of $89 \%$ (99\%), 94\% (98\%), and $97 \%(93 \%)$. The authors of this research work also include a variable kappa, a statistical metric that compares the assessment of the alignment between ML and clinicians' results. The kappa coefficient ranges from 0.90 to 0.91 , which means an almost perfect model.

Other research related to ventilation carried out by authors in [30] have pointed out the need for developing promising methods based on machine learning techniques for prolonged mechanical ventilation situations and potential complications. Research was focused on reaching improvements in areas such as:

- prediction of arterial blood gases,

- successful extubation,

- spontaneous breathing trails, and

- development of autonomous ventilator setting adjustments.

A common difficulty for all this research work is the lack of data sources necessary for reducing bias and getting better results supported by more clinical cases. This fact has become more acute when considering the COVID-19 crisis.

\section{B. Image Processing For the Diagnosis of COVID-19}

ML has been used for the detection, segmentation and classification of medical imaging. Some examples of it are:

- The analysis of histological sections of tumors for detecting the presence or absence of metastases,

- the application on radiological images in order to segment different areas, and

- the categorization of diagnostics based on the classification of images.

In regard to COVID-19 and IP, one of the main problems is the similarity of symptoms related to bacterial and viral pneumonia in chest radiographies. In this way, deep learning can be applied to detect and differentiate clinical nuances. According to the Treatment Guidance 
for Critically Ill Patients with COVID-19 from Massachusetts General Hospital [12], one of the clinical futures that characterizes this condition is chest abnormalities, including bilateral opacities. These abnormalities are due in many cases to bacteria that were acquired during pneumonia and viral pneumonia outbreaks. In fact, both conditions can generate confusion, particularly in those with severe illness requiring admission to ICU and mechanical ventilation. In order to differentiate between COVID-19 induced pneumonia and other types of pneumonias, there are some distinctive trends and distribution of CTs (Table V) evidences that could be taken into account [31] in order to apply ML techniques for isolating COVID-19 diagnosis:

- Ground glass opacification (GGO) (88.0\%),

- Bilateral involvement (87.5\%),

- peripheral distribution $(76.0 \%)$,

- and multilobar (more than one lobe) involvement (78.8\%).

TABLE V. PAtterns And Distribution of CT Studies

\begin{tabular}{ccc}
\hline Imaging Insights & $\begin{array}{c}\mathrm{N}^{\circ} \\
\text { Studies }\end{array}$ & $\begin{array}{c}\mathrm{N}^{\circ}(\%) \text { of registered cases/ } \\
\text { total } \mathrm{n}^{\circ} \text { of patients }\end{array}$ \\
\hline Bilateral involvement & 12 & $435 / 407(87.5)$ \\
Peripheral distribution & 12 & $92 / 121(76.0)$ \\
Posterior involvement & 1 & $41 / 51(80.4)$ \\
Multilobar involvement & 5 & $108 / 137(78.8)$ \\
Ground-glass opacification & 22 & $346 / 393(88.0)$ \\
Consolidation & 10 & $65 / 204(31.8)$ \\
\hline
\end{tabular}

Most usual patterns and distribution on initial CT Studies of 919 Patients with Coronavirus Disease (COVID-19).

Nevertheless, authors in [32] suggest that daily chest X-rays have no positive effect over the outcome in the ICUs once COVID-19 has been diagnosed. These scientific study results are aligned with the IP and AI diagnostic techniques results. Medical images reduce the over exposure to radiation and are more predictive during the first stages of the condition.
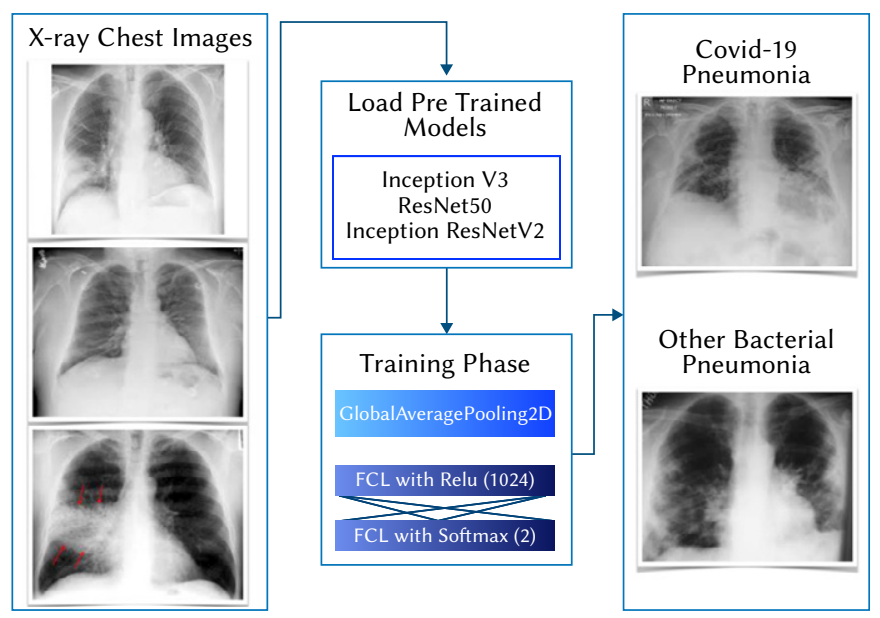

Fig. 10. Schematic representation of the pre-trained models to predict COVID-19 by comparing patients with and without the disease. Model adapted from the figure published [22].

In this way authors in [22] have recently detected COVID-19 by applying IP and convolutional neural networks (CNNs) techniques to the patients' X-ray images. Moreover, this approach is more relevant if we consider that results are more accurate than the "gold standard" for COVID-19 diagnosis, and that the RT-PCR does not have enough sensitivity for diagnosis purposes. This means that many patients could not receive adequate treatment. Authors in [22] propose using a pre-trained neural network (ResNet50, InceptionV3 and InceptionResNetV2) processing applied to X-ray images as these are faster and get accurate diagnoses with better outcomes than PCR. Fig. 10 shows a schematic representation of this network comparing X-rays of patients with COVID-19 and those of patients without the disease. The images dataset available is small (100 images in total) so the results should be interpreted with caution. The results of the study show that with this dataset the ResNet50 network has the best performance, with an accuracy of $98 \%$.

Fig. 11 shows the accuracy results coming from the implementation of ResNet50, InceptionV3 and Inception-ResNetV2, which try to make predictions about patients with COVID-19 based on X-ray images. The best accuracy curve model has been obtained by ResNet50.

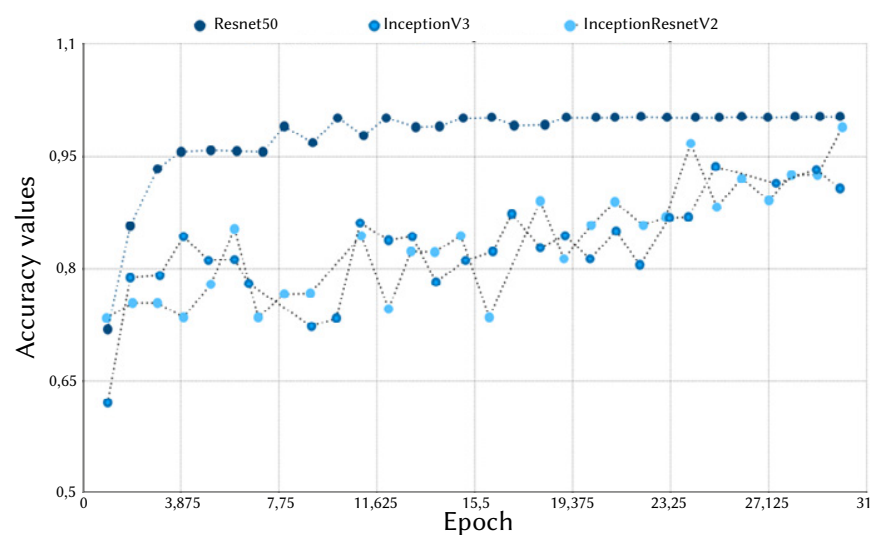

Fig. 11. Authors have used three pre-trained models (ResNet50, InceptionV3 and Inception resNetV2) to make predictions of coronavirus patients based on chest X-ray images. This figure shows the accuracy curve for each model. In this case, the highest accuracy has been reached with ResNet50. Model adapted from the figure published in [22].

Authors in [33] applied a deep learning system to diagnose pneumonia in COVID-19 patients. However, in this case, the research was conducted with CT images used for diagnostic purposes. The research work follows the same argument that authors in [32] made, which is that there is a low positive rate in early stages of COVID-19 obtained from PCR. In this case, the dataset was bigger than the one used in [22], which sum a total of 618 CTs from 110 patients with COVID-19 and 224 patients with Influenza-A pneumonia. In this case, the accuracy was $86.7 \%$. Even with these promising results, this technique is relatively questionable due to use of CTs for routine diagnostic purposes. In terms of health care system efficiency, CTs are not a kind of medical equipment that could be used in situation of ICU and ER collapse. Moreover, CT rooms could lead to a high percentage of viral transmission if these rooms are crowded.

Authors in [4] published in March 2020 a COVID-19 study applying a combination of deep learning architectures (AlexNet, VGG16, VGG19, GoogleNet, ResNet18, ResNet50, ResNet101, InceptionV3, InceptionResNetV2, DenseNet201 and XceptionNet) for extracting relevant features that were used for feeding an SVM classifier. In this study, authors have considered not only patients suffering from COVID-19 but also SARS and MERS. This approach involves similar diseases but with some specific characteristics that present differences among groups. The comparative study (see Table VI) shows that ResNet50 model had the best result, compared to the others. However, the dataset with X-ray images is relatively small to be able to conclude that this system can work properly in real time.

A different approach used the COVID-Net convolutional neural network model [34]. This research project has been proposed as a 
chest X-Ray image screening method. This ML tool can be considered as a faster alternative to PCR or at least, as a complement to this in vitro diagnosis method. Moreover, this network has been pre-trained with the ImageNet dataset [35] in order to get better results. After this pre-trained process, the network has been trained with a new dataset COVIDx that comprises 16,756 chest images from 13,645 COVID-19 patients. The COVIDx dataset groups:

- the COVID-19 image dataset [36]. This dataset contains frontal view X-rays images and is the greatest public database for COVID-19 image and prognostic data, and

- the Radiological Society of North America (RSNA) Pneumonia Detection Challenge Dataset [37] is a subset of 30,000 chest radiograph (CXR) exams taken from the NIH CXR14 dataset [38]. In accordance with the whole group of exams, 15,000 exams had positive results for pneumonia or similar pathological conditions such as consolidation and infiltrate.

TABle VI. The Same Letter(s) Within A column Means That Results Are Not Statically Significant $(P=0.05)$ According to Duncan's Multiple Range Test (SPSS Version 26)

\begin{tabular}{cccc}
\hline \hline Classification Model & Accuracy & Sensitivity & Specificity \\
AlexNet & $.933235 \mathrm{~d}$ & $.934117 \mathrm{~b}, \mathrm{c}$ & $.932352 \mathrm{c}$ \\
DenseNet201 & $.938823 \mathrm{~d}$ & $.943529 \mathrm{c}, \mathrm{d}$ & $.934117 \mathrm{c}$ \\
GoogleNet & $.914411 \mathrm{~b}, \mathrm{c}$ & $.898235 \mathrm{a}$ & $.930588 \mathrm{~b}, \mathrm{c}$ \\
Inceptionv3 & $.910882 \mathrm{~b}$ & $.911176 \mathrm{a}, \mathrm{b}$ & $.910588 \mathrm{~b}, \mathrm{c}$ \\
ResNet18 & $.910882 \mathrm{~b}$ & $.911176 \mathrm{a}, \mathrm{b}$ & $.910588 \mathrm{~b}, \mathrm{c}$ \\
ResNet50 & $.953823 \mathrm{c}$ & $.972941 \mathrm{~d}, \mathrm{c}$ & $.934705 \mathrm{c}$ \\
ResNet101 & $.892647 \mathrm{a}$ & $.912352 \mathrm{a}, \mathrm{b}$ & $.872941 \mathrm{a}$ \\
VGG16 & $.927647 \mathrm{c}, \mathrm{d}$ & $.970745 \mathrm{c}$ & $.880588 \mathrm{a}$ \\
VGG19 & $.929117 \mathrm{~d}$ & $.951176 \mathrm{c}, \mathrm{d}, \mathrm{c}$ & $.907058 \mathrm{~b}$ \\
XceptionNet & $.939117 \mathrm{~d}$ & $.947647 \mathrm{c}, \mathrm{d}, \mathrm{c}$ & $.930588 \mathrm{~b}, \mathrm{c}$ \\
Inceptionresnetv2 & $.933235 \mathrm{~d}$ & $.852941 \mathrm{c}, \mathrm{d}, \mathrm{c}$ & $.913529 \mathrm{~b}, \mathrm{c}$
\end{tabular}

Statistical analysis for SVM models based on features extracted from different CNN models

COVID-Net's results offer three possibilities: non-infection, COVID-19 viral infection and other non-COVID-19 infection. This methodology helps clinicians to stratify patients in an easy way and decide which is the most adequate treatment for each patient. COVIDNet has achieved a $92.6 \%$ of accuracy. This percentage is very relevant for taking into consideration the complexity of the ICUs. At the same time, results regarding its sensitivity (Table VII) are quite good with respect to epidemiological requirements such as the limitation over missed COVID-19 cases.

TABLE VII. COVID-Net Sensitivity (\%)

\begin{tabular}{ccc}
\hline Normal & Non-COVID19 & COVID-19 \\
97.0 & 90.0 & 87.1 \\
\hline
\end{tabular}

Sensitivity effectiveness related to COVID-Net results

\section{ML Biomarker Processing For COVID-19}

The COVID-19 pandemic has been a one in a century event, whose symptoms are fever, cough, dyspnea, and viral pneumonia. For this reason, diagnostic tests have become very useful for confirming suspected cases, screening patients, and conducting virus surveillance. Currently, the gold standard technique that is used to detect COVID-19 is the PCR test, which is based on the diagnostic protocol provided by the WHO on 13 January 2020 [39]. Fig. 12 shows the steps that are part of the process [40] for identifying active COVID-19 infection in a patient. The whole process consists in three steps:

1. The first one consists in taking a sample from the patient's nose or mouth.

2. After that, the second step continues with a RNA isolation from a patient's sample.

3. The third and final step is the conversion of the RNA into complementary DNA (cDNA) using an enzyme called reverse transcriptase. This component is then re-loaded into a plate and place in a PCR machine. After that the results from the PCR machine are evaluated in a computer. If the level of COVID-19 RNA in the sample is over the threshold, the diagnosis is confirmed, and the patient is positive for COVID-19.

However, COVID-19 patients frequently present additional findings in laboratory testing, which are carried out before ICU admission. ML has been commonly used for the interpretation of data in different fields of biomedicine such as genomics, transcriptomics and proteomics pathways [16]-[17]. At the same time, it has also been used in biological materials in clinical laboratory medicine for identifying biomarkers. Typically, the ML approach is based on rules that are learned by the machine. In general terms, a discussion of rules is not appropriate because predictions are based on non-linear parameters that hide decision-making around the data.

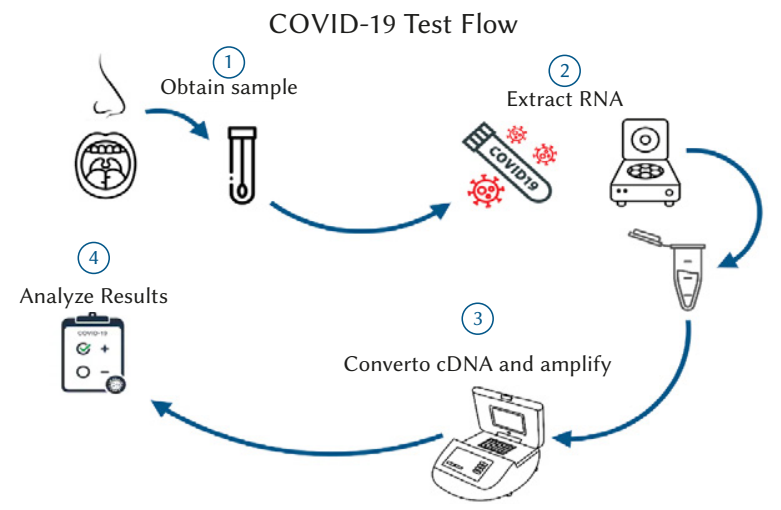

Fig. 12. Workflow for identifying patients with active COVID-19 infection. Model adapted from the figure published [40].

In reference to COVID-19, authors in [41] have found some clinical biomarkers that present abnormal values in the white blood cell and absolute lymphocyte count. In order to isolate which of them should be considered as survival predictors, AI algorithms analyze all these biomarkers, in combination with other clinical findings that can be analyzed. In this way, authors in [42], have applied ML models (XGBoost) by selecting three biomarkers from the Electronic Health Record System EHRS: Lactic Dehydrogenase (LDH), Lymphocyte and High-sensitivity C-Reactive Protein (hs-CRP).

The training and test datasets are composed of 1,523 blood samples with complete measures coming from 375 patients. Additionally, the project adds 228 more blood samples for the validation set of 29 patients. Survival prediction has been about $90 \%$. From the point of view of clinical decisions, the biomarkers selected by the algorithm described in Fig. 13 make sense because:

- The increase in Serum LDH is associated with a prognostic marker of lung injury.

- hsCRP could identify risk of death since its increase is associated with ARDS prognosis and inflammation.

- Lymphocyte associated Lymphopenia is common in COVID-19 patients with severity and mortality. 


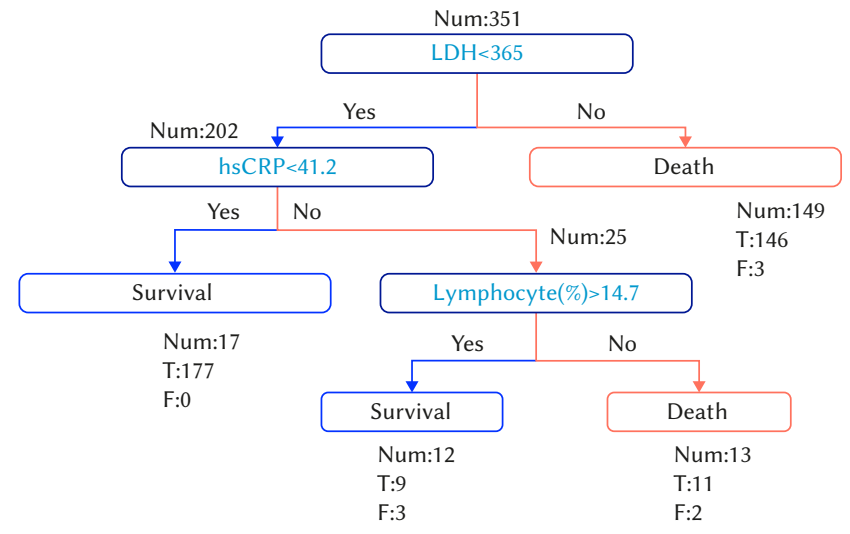

Fig. 13. Based on Yan et al. results, the best performing tree based discover three (LDH, hs-CRP and Lymphocyte) key features and their thresholds values over a population of 351 patients with the aim of being able to identify high risk patients before an irreversible lesion occur. Model adapted from the figure published [42].

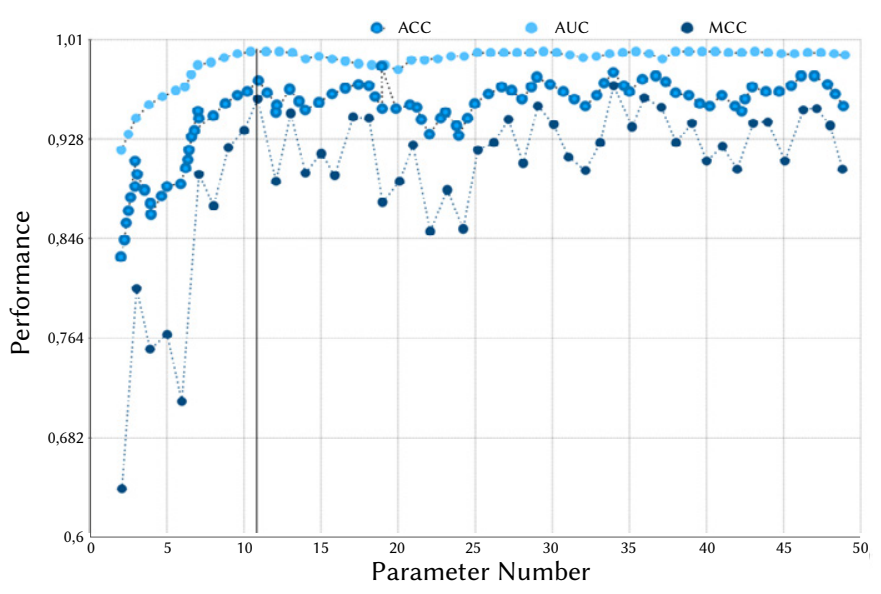

Fig. 14. Model performance: ACC (0.98), MCC (0.95) and AUC (1.0). Model adapted from the figure published [43].

Authors in [43] have developed a tool to triage suspected COVID-19 patients. The accuracy has been $97.95 \%$ and $96,97 \%$ for the cross-validation set and test set, respectively. However, the model has been also assessed with an external validation obtaining sensitivity, specificity, and overall accuracy of $95.12 \%, 96,97 \%$ and 95.95\%, respectively. The predictive model has been conducted based on Random Forest algorithm (RF), which has been able to identify 11 laboratory parameters: Total Proteins, Glucose, Calcium, Creatine Kinase Isoenzyme, Magnesium, Basophil, Total bilirubin, Creatinine, Lactate Dehydrogenase, Kalium and Platelet Distribution. These results (see Fig. 14) could simplify the laboratory blood processes and at the same time, reduce the time scope for applying treatments in COVID-19 patients and improve the disease monitoring.

\section{CONCLUSIONS}

COVID-19 has shown the need for new clinical pathways focused on prevention, as well as a need for improvements in methods and processes for diagnosis, prognosis and treatment. This pandemic has also had a huge impact on the generation of associated clinical and research data. An important part of these datasets has been generated in ICU and ER rooms. The new illness in many countries has led to or surpassed the collapse in health care systems, due to a shortage of intensive care professionals and a high demand for ICU equipment. Similar situations have been related to compassionate use of drugs that had not been previously approved for specific COVID-19 clinical use. This unprecedented scenario requires a cross clinical and technical disciplinary approach to tackle the pandemic. Moreover, when it is necessary to take into account patient data, its automatic and centralized management is beneficial. For this reason, COVID-19 patient care will require even more automatic integration and analysis of multiple parameters and data sources.

In this situation computational clinical tools based on AI systems have a huge impact on improvements for dealing with COVID-19 patients. The next goals will focus on respiratory status monitoring in real time, the design of new therapeutic treatments for SARS-CoV-2, hemodynamic management, imaging and laboratory research, as well as reducing possible delays in patient admission and optimizing the clinician's time. Notwithstanding, we must not forget and remember that ML systems are in place to support and not replace the clinician.

\section{REFERENCES}

[1] World Health Organization. (2020). WHO statement regarding cluster of pneumonia cases in Wuhan, China. Beijing: WHO, 9.

[2] C. Anastassopoulou, L. Russo, A. Tsakris, C. Siettos. Data-based analysis, modelling and forecasting of the COVID-19 outbreak. PloS one, vol. 15, no. 3, pp. e0230405, 2020.

[3] A. Alimadadi, S. Aryal, I. Manandhar, P. B. Munroe, B. Joe, X. Cheng. Artificial Intelligence \& Machine Learning to Fight COVID19. 2020.

[4] P. K. Sethy, S. K. Behera. Detection of coronavirus Disease (COVID-19) based on Deep Features, 2020.

[5] F.A. Murphy. Emerging zoonoses. Emerg Infect Dis. Vol. 4, no. 3, pp. 429-435, 1998.

[6] M.A. Shereen, S. Khan, A. Kazmi, N. Bashir R. Siddique. COVID-19 infection: Origin, transmission, and characteristics of human coronaviruses. Journal of Advanced Research, 2020.

[7] G. Venkatesan, V. Balamurugan, P. N. Gandhale, R. K. Singh, V. Bhanuprakash. Viral zoonosis: a comprehensive review. Asian Journal of Animal and Veterinary Advances, vol. 5, no. 2, pp. 77-92, 2010.

[8] S. Wan, Y. Xiang, W. Fang, Y. Zheng, B. Li, Y. Hu, X. Huang. Clinical features and treatment of COVID-19 patients in northeast Chongqing. Journal of medical virology. 2020.

[9] T. Klopfenstein, L. Toko, P. Royer, Q. Lepiller, V. Gendrin, S. Zayet Features of anosmia in COVID-19. Médecine et Maladies infectieuses, 2020.

[10] World Health Organization. Clinical management of COVID-19: interim guidance, 27 May 2020 (No. WHO/2019-nCoV/clinical/2020.5). World Health Organization.

[11] L. A. Vaira, G. Salzano, G. Deiana, G. De Riu. Anosmia and ageusia: common findings in COVID-19 patients. The Laryngoscope, 2020.

[12] Massachusetts General Hospital Treatment Guidance for Critically Ill Patients w/ COVID-19. https://bit.ly/mghcovid. Last accessed July 2020.

[13] H. A. Rothan, S. N Byrareddy. The epidemiology and pathogenesis of coronavirus disease (COVID-19) outbreak. Journal of autoimmunity, 102433, 2020.

[14] R. Li, C. Rivers, Q. Tan, M. B. Murray, E. Toner, M. Lipsitch. Estimated Demand for US Hospital Inpatient and Intensive Care Unit Beds for Patients With COVID-19 Based on Comparisons With Wuhan and Guangzhou, China. JAMA network open, vol. 3, no. 5, pp. e208297-e208297, 2020.

[15] P. Gautret, J. C. Lagier, P. Parola, L. Meddeb, M. Mailhe, B. Doudier, S. Honoré. Hydroxychloroquine and azithromycin as a treatment of COVID-19: results of an open-label non-randomized clinical trial. International journal of antimicrobial agents, 105949, 2020.

[16] B. Cao, Y. Wang, D. Wen, W. Liu, J. Wang, G. Fan, X. Li. A trial of lopinavir-ritonavir in adults hospitalized with severe Covid-19. New England Journal of Medicine, 2020

[17] M. Wang, R. Cao, L. Zhang, X. Yang, J. Liu, M. Xu, G. Xiao. Remdesivir and chloroquine effectively inhibit the recently emerged novel coronavirus (2019-nCoV) in vitro. Cell research, vol. 30, no. 3, pp. 269-271, 2020.

[18] P. Simon. Too Big to Ignore: The Business Case for Big Data. (Vol. 72). 
John Wiley \& Sons, 2013.

[19] G. Gutierrez. Artificial Intelligence in the Intensive Care Unit. Critical Care, vol. 24, article number 101, pp. 1-9, 2020.

[20] G. S. Randhawa, M. P. Soltysiak, H. El Roz, C. P. de Souza, K. A. Hill, L. Kari. Machine learning using intrinsic genomic signatures for rapid classification of novel pathogens: COVID-19 case study. Plos one, vol. 15, no. 4, pp. e023239, 2020.

[21] S. Wang, B. Kang, J. Ma, X. Zeng, M. Xiao, J. Guo, B Xu. A deep learning algorithm using $\mathrm{CT}$ images to screen for Corona Virus Disease (COVID-19). MedRxiv, 2020.

[22] A. Narin, C. Kaya, Z. Pamuk. Automatic detection of coronavirus disease (COVID-19) using X-ray images and deep convolutional neural networks. arXiv preprint arXiv:2003.10849, 2020.

[23] F.A. Saiz, I. Barandiaran. COVID-19 Detection in Chest X-ray Images using a Deep Learning Approach. International Journal of Interactive Multimedia and Artificial Intelligence, vol 6. n. 12, pp. 11-15, 2020.

[24] F. Cabitza, G. Banfi. Machine learning in laboratory medicine: waiting for the flood? Clinical Chemistry and Laboratory Medicine (CCLM), vol. 56, no. 4, pp. 516-524, 2018.

[25] N. Ferguson, D. Laydon, G. Nedjati, N. Imai, K. Ainslie, M. Baguelin, A. Dighe. Impact of non-pharmaceutical interventions to reduce COVID19 mortality and healthcare demand, 2020.

[26] P. Richardson, I. Griffin, C. Tucker, D. Smith, O. Oechsle, A. Phelan, J. Stebbing. Baricitinib as potential treatment for 2019-nCoV acute respiratory disease. Lancet (London, England), vol. 395. No. 10223, pp. e30-e31, 2020.

[27] M. J. Tobin. Mechanical Ventilation. New England Journal of Medicine, vol. 330, no. 15, pp. 1056-1061, 1994.

[28] L. Blanch, B. Sales, J. Montanya, U. Lucangelo, O. Garcia-Esquirol, A. Villagra, G Murias. Validation of the Better Care system to detect ineffective efforts during expiration in mechanically ventilated patients: a pilot study. Intensive Care Medicine, vol. 38, no. 5, pp. 772-780, 2012.

[29] B. Gholami, T.S. Phan, W.M. Haddad, A. Cason, J. Mullis, L. Price, J.M. Bailey. Replicating human expertise of mechanical ventilation waveform analysis in detecting patient ventilator cycling asynchrony using machine learning, Computers in Biology and Medicine, 2018.

[30] M. T. Kwong, G. W. Colopy, A. M. Weber, A. Ercole, J. H. Bergmann. The efficacy and effectiveness of machine learning for weaning in mechanically ventilated patients at the intensive care unit: a systematic review. Bio-Design and Manufacturing, vol. 2, no. 1, pp. 31-40, 2019

[31] S. Salehi, A. Abedi, S. Balakrishnan, A. Gholamrezanezhad. Coronavirus disease 2019 (COVID-19): a systematic review of imaging findings in 919 patients. American Journal of Roentgenology, pp. 1-7, 2020.

[32] Y. Oba, T. Zaza. Abandoning daily routine chest radiography in the intensive care unit: meta-analysis. Radiology, vol. 255, no. 2, pp. 386-395, 2010.

[33] X. Xu, X. Jiang, C. Ma, P. Du, X. Li, S. Lv, Y. Li. Deep learning system to screen coronavirus disease 2019 pneumonia. arXiv preprint, 2020.

[34] L. Wang, A. Wong. COVID-Net: A Tailored Deep Convolutional Neural Network Design for Detection of COVID-19 Cases from Chest X-Ray Images. arXiv preprint arXiv:2003.09871, 2020.

[35] J. Deng, W. Dong, R. Socher, L. Li, K. Li, L. Fei-Fei. Imagenet: A large-scale hierarchical image database. In 2009 IEEE conference on computer vision and pattern recognition, pages 248-255, 2009.

[36] J. P. Cohen, P. Morrison, L. Dao, K. Roth, T. Duong, M. Ghassemi. COVID-19 Image Data Collection: Prospective Predictions Are the Future. arXiv preprint, 2020.

[37] X. Wang, Y. Peng, L. Lu, Z. Lu, M. Bagheri, R. Summers. ChestX-ray8: Hospital-scale Chest X-ray Database and Benchmarks on Weakly Supervised Classification and Localization of Common Thorax Diseases. In Proceedings of the IEEE conference on computer vision and pattern recognition (pp. 2097-2106), 2020.

[38] Radiological Society of North America. RSNA pneumonia detection challenge. https://bit.ly/kagglersna. Last accessed July 3, 2020.

[39] V. M. Corman, O. Landt, M. Kaiser, R. Molenkamp, A. Meijer, D. K. Chu, D. G. Mulders. Detection of 2019 novel coronavirus (2019-nCoV) by realtime RT-PCR. Eurosurveillance, vol. 25, no. 3, pp. 2000045, 2020.

[40] Explained: how to identify active covid-19 infection in people. https:// www.researchamerica.org/blog/explained-how-identify-active-covid19-infection-people by 06/07/2020. Last accessed July 3, 2020.
[41] M. Arentz, E. Yim, L. Klaff. Characteristics and Outcomes of 21 Critically Ill Patients With COVID-19 in Washington State. JAMA. Published online March 19, 2020.

[42] L. Yan, H. T. Zhang, J. Goncalves, Y. Xiao, M. Wang, Y. Guo, X. Huan. A machine learning-based model for survival prediction in patients with severe COVID-19 infection. medRxiv, 2020.

[43] J. Wu, P. Zhang, L. Zhang, W. Meng, J. Li, C. Tong, M. Zhao. Rapid and accurate identification of COVID-19 infection through machine learning based on clinical available blood test results. medRxiv, 2020.

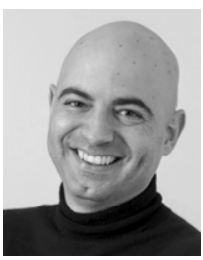

\section{Sergio Muñoz Lezcano}

Sergio Muñoz Lezcano is a full-time innovation and digital health manager at the Spanish Federation of Healthcare Technology Companies. Actually, he is a PhD Student at the Universidad Internacional de La Rioja (UNIR) involved in artificial intelligence research applied in healthcare focus on intensive care units (with emphasis on sepsis condition in adults and neonates). He obtained a MSc in Artificial Intelligence in 2019 at UNIR and MBA in 2000 at ICADE - Universidad Pontificia Comillas. He is also a recognized business mentor working with EU entrepreneurship and startups programs in health care ecosystems and coordinates Spanish innovation Platform in Health Technology.

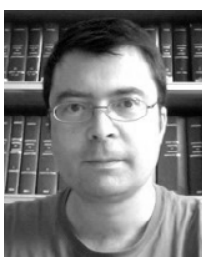

\section{Fernando Carlos López Hernández}

Fernando Carlos López Hernández is a full-time associate professor at the Universidad Internacional de La Rioja (UNIR). His current research interests lie in image processing, computer vision, statistical machine learning and data-driven science. He is the education manager of the Doctorate Program in Computer Science, and a of Course of Robotics for Education. In addition, he works as a lecturer in the Computer Science degree (Statistics, Algebra, Discrete Mathematics, Algorithms Complexity, Image Processing, Signal Processing, Computer Graphics, Compilers). Until 2015, he was the director, education manager as well as a lecturer in the Master in Mobile Apps degree (iOS technologies) at UNIR. Before joining UNIR, he worked for the Universidad Autónoma de Madrid (UAM) as a post-doctoral researcher in European research projects. He obtained his EU-wide recognized $\mathrm{PhD}$ degree in Computer Science and Telecommunications in 2010 at the Video Processing and Understanding Lab (VPU Lab) of the UAM, a period during which he worked in 2 European research projects related to his thesis.

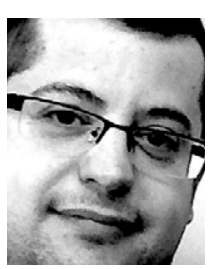

Alberto Corbi Bellot

Alberto Corbi Bellot is a senior researcher at the Research Institute for Innovation \& Technology in Education (iTED) and as an assistant professor at the Engineering School, which are both part of the Universidad Internacional de La Rioja (UNIR). He is currently involved in a variety of research fields: e-learning standards, systems interoperability, medical physics, radiological protection,

STEM education, the monitoring of physical activities, the social implications of technology (with emphasis on social networks), e-health advancement (with emphasis on Alzheimer's disease characterization and clinical information standards) and environmentalism. He has published over 20 research papers on all these subjects, and he is a speaker and knowledge disseminator at radios, podcasts, scientific workshops, magazines, academic settings, and outreach events. 